\title{
Differential topology method of identifying 3D null points in solar vector magnetic fields
}

\author{
Hui Zhao, Jingxiu Wang, Jun Zhang \\ and Chijie Xiao
}

National Astronomical Observatories, Chinese Academy of Sciences, Beijing 100012, China email: zhaohui@ourstar.bao.ac.cn

\begin{abstract}
Employing the Poincaré index of isolated null-point in a vector field, we work out a mathematical method of searching 3D null-points in coronal magnetic fields. After introducing the theory of differential topology, we test the method by using the analytical model of Brown $\&$ Priest. The location of null-point identified by our method coincides precisely with the analytical solution. Finally we apply the method to search 3D null-points in coronal magnetic fields reconstructed based on observed magnetograms of two active regions (NOAA AR 10488 and AR 10720). We find that the 3D null-points seem to be a key element to the magnetic topology associated with flare occurrence.
\end{abstract}

Keywords. Sun: solar flare, magnetic topology, 3D null-point

\section{Introduction}

It has been widely accepted that the topological structures of solar magnetic fields are closely associated with magnetic reconnection and hence solar flares and coronal mass ejections (Sweet, 1969). To understand the magnetic topological structures of the corona, 3D null-points, which appear a vital topological feature in the magnetic fields, should be thoroughly investigated. The definition, classification and properties of the null-point have been described by Lau \& Finn (1990). In analytical condition Lau (1993), Brown \& Priest (2001), respectively, presented the null-points and associated topological structures in their models. Comparing the topological sketches and observations, Filippov (1999), Aulanier et al. (2000) and Fletcher et al. (2001) identified the null-points in corona. A general and direct method, independent of analytical models and topology sketches, has been suggested by Wang \& Wang (1996) in 2D vector magnetic fields. Inspired by this work, we develop a method (from Poincaré-Hopf theorem in differential topology) which can identify 3D null-points directly in any given 3D vector fields.

\section{Theory and Method}

Suppose $V(M)$ is a continuous vector field in space $M, p \in M$ satisfies: $V(p)=0$ and there exits a neighborhood $U$ of $p, V(U \backslash\{p\}) \neq 0$. We say $p$ is a isolated singular point(or null-point) in $V(M)$. In an actual vector field, we can not expect such a null-point locate at the very pixel which we were selecting. Therefore we need a method by which we can identify the null-point through studying the behaviors of vectors in its neighborhood. In global differential topology, the Poincaré index can meet with this requirement.

In two dimension space, we select a close curve to surround the point we detect. For example, $M=S^{1}$ is the close curve, and its positive orientation is anti-clockwise (Fig. 1 left). The vector field on $M$ is $V=V(M)$. Displace these vectors $(V(M))$ to origin and normalize them, we get a continuous mapping $f$ from $M$ to $N=S^{1}$. Along $M, f(M)$ 

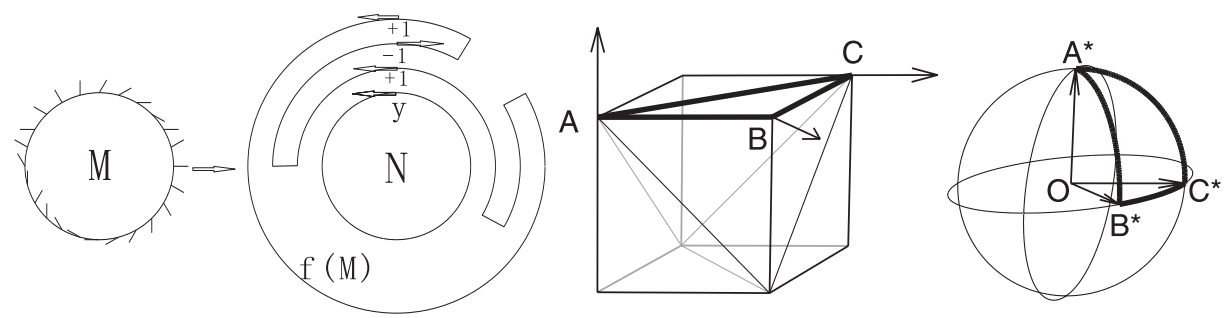

Figure 1. The illustration of theory in 2D condition. Vectors on $M$ are shown in short lines (left). Illustration of theory in 3D condition. Vectors at $A, B$ and $C$ on cube vertex and their images $A^{*}, B^{*}$ and $C^{*}$ on $S^{2}$ are shown in arrows. $\triangle A B C$ and corresponding spherical triangle are marked by bold sidelines (right).

might go to and fro in $N$. For seeing more clearly, we extend $f(M)$ in radial direction outside $N$. The Poincaré index is the number of how many times does the $f(M)$ rotate around $N$ in positive direction. If index does not equal to zero, there must be a null-point in the region surrounded by $M$. It is alike in 3D condition. In practice, we choose a cube as the closed surface include a singular point (Fig. 1 right). Normalize the vector field on the cube surface and displace the image on $S^{2}$, integrate these spherical triangles and divided the integration by $4 \pi$. Then we get the index which tell us the image $(f(M))$ wraps $S^{2}$ how many times in positive orientation.

\section{Application}

\subsection{Test in an analytical model}

We first use our method to search a known null-point of the analytical model suggested by Brown \& Priest (2001). There exist three negative sources of equal magnetic strength, $\epsilon_{S}=-1$, at $(0,1,0),\left(\cos \frac{7}{6} \pi, \sin \frac{7}{6} \pi, 0\right),\left(\cos \frac{11}{6} \pi, \sin \frac{11}{6} \pi, 0\right)$ and one positive source of unit strength in the center of the triangle. The analytical solution gives a null-point at $(0,0,0.962)$. In the box of $[-2,2] \times[-2,2] \times[0,3]$, we sum up the values of 12 spherical triangles in each little cube (Fig. 1 right) composed by 8 pixels. If a cube has no any singular point, its index should be equal to zero. In the box we find the only one cube which index is equal to -1 includes the null-point that we have known by the analytical solution, while for others the indexes are all equal to zero.

\subsection{Applying to an active region}

We also applied the method to search for null-points in extrapolated magnetic fields of solar active regions (ARs). NOAA 10488 is one of the three super-active regions appeared in the interval between October 18 and November 4 of 2003. These three ARs produced many unexpected eruptive events (Zhang et al. 2003). We use linear force-free extrapolation method with a constant $\alpha$ suggested by Seehafer (1978) to get 3D vector magnetic fields. The boundary condition is taken from MDI magnetogram and the value of $\alpha$ is obtained from the Huairou vector magnetograms at the nearest time. We expectably find a null-point above this AR. Figure 2 shows that the field lines near this null-point are very much similar to the analytical model of Brown \& Priest. Some magnetic lines of force which anchored within two flare ribbons are passing the vicinity of the 3D nullpoint or have some relations with the spine and fan structures associated with the null point (Fig. 2). Different from the analytical model, the spine is a curve from one positive source to another positive source, while the fan structure is dome-like, but asymmetric. The null-point, where the spine cross through the dome, is at the height of $12^{\prime \prime}(8700 \mathrm{~km})$ above the solar photosphere. 


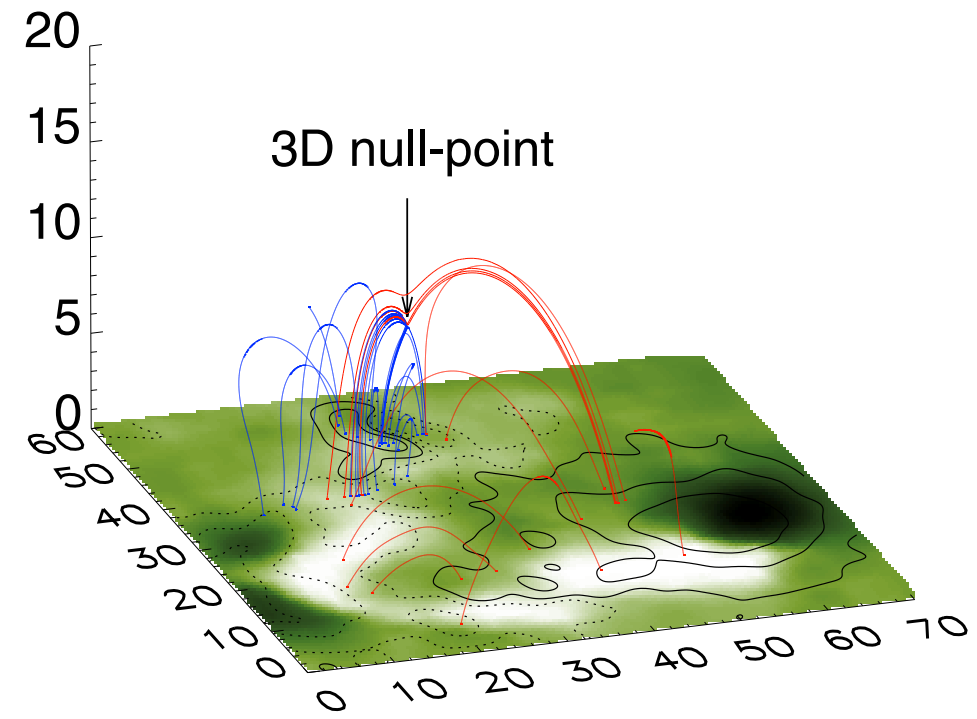

Figure 2. The linear force-free field lines, reconstructed with the MDI magnetogram of 2003 Oct $29,22: 23$ as the extrapolation boundary. Units are MDI pixels(about $2^{\prime \prime} .0$ ) The H $\alpha$ image of Huairou places on the $z=0$ plane. And the contour on the $z=0$ plane is longitudinal magnetism. The dashed curves are negative contours, the solid curves are positive contours. The spine is a curve form one positive source to another positive source. A dome-like surface divided the region $z>0$ into two parts marked with two colors. A 3D null point exists at $(31.2,47.4,6.2)$ through which the spine cross the dome.

NOAA 10720 is another super-active regions appeared in the interval between January 11 and January 23 of 2005 . It produced two X class flares on January 15 . We use quasilinear force-free extrapolation method suggested by Wang et al. (2001) to get 3D vector magnetic fields. The boundary condition is taken from BBSO vector magnetogram. Above this AR we find several null-points (Fig. 3). Each topology marked with different color is composed with spine and fan structure as in case of NOAA 10488 and analytical model. We find green field lines coil round its null-point on the fan-like singular surface. The spines of pink and brown field lines become the boundary of green fan. The blue and red topologies is partitioned by green fan. The arch made of green, pink and brown field lines locates at the flare ribbons.

\section{Discussion and Conclusion}

Undoubtedly null-point in the corona is a vital topological feature for understanding coronal phenomena. In this work we present theoretical conception and method of identifying the 3D null-points in vector fields. As a test we use this method to search a known 3D null-point in an analytical model of Brown \& Priest. By the application to AR 10488 and AR 10720 we find solar flares are quite possibly related to 3D null-points (pointed out by arrows). For comprehensively studying the relations of magnetic topology and solar activity, we should detect the singular lines and singular surfaces in further work. Surely we should also track these topology features for fully illustrating the time dependent magnetic topology related to which physical process of coronal phenomena. 


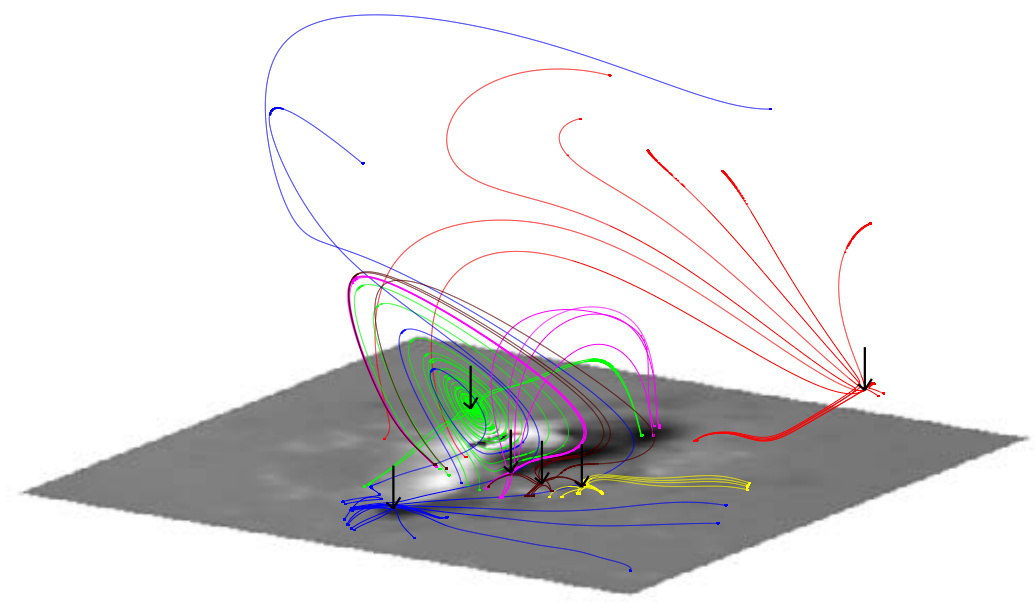

Figure 3. The linear force-free field lines, reconstructed with the BBSO vector magnetogram of 2005 Jan 15, 21:51 as the extrapolation boundary. The $\mathrm{H} \alpha$ image of BBSO places on the $z=0$ plane. Different magnetic topologies composed of spine and fan are marked with different colors. The null-points are point out by arrows.

\section{Acknowledgements}

The work is supported by the National Natural Science Foundation of China (10233050) and National Key Basic Research Science Foundation (G2000078404).

\section{References}

Aulanier, G., DeLuca, E.E., Antiochos, S.K., McMullen, R.A., \& Golub, L., 2000, APJ, 540, 1126.

Brown, D.S., Priest, E.R., 2001, A\&A, 367, 339.

Filippov, B., 1999, Solar Phys., 185, 297.

Fletcher, L., Metcalf, T.R., Alexander, D., et al. 2000, Apj, submitted.

Lau, Y.T., 1993, Solar, Phys., 148, 301.

Lau, Y.T., Finn, J.M., 1990, APJ, 350, 672.

Seehafer, N., 1978, Solar Phys., 58, 215.

Sweet, P.A., Nuovo Cimento Suppl.8, Ser.X, 188.

Sweet, P.A., 1969, ARA\&A, 19, 163.

Wang, H.N., Wang, J.X., 1996, A\&A, 313, 285.

Wang, H.N., Yan, Y.H., Sakurai, T., 2001, Solar Phys., 201, 323. 